NSF-ITP-90-63

PURD-TH-90/12

Fermilab-Pub-90/92-T

\section{On the Scaling Properties of Quenched QED}

Williarn A. Bardeen ${ }^{(1)}$, Sherwin T. Love'2) and Vtadimir A. Miransky'31

Institiute for Theoretical Physics

University of California

Santa Barbara, CA 93106

\section{Abstract}

Critical scaling laws are studied in quenched quantum electrodynamics with induced four fermion interactions that drive the theory to criticality. The critical exponents are calculated in the quenched, planar model and the physical picture extracted is consistent with recent results from lattice simulations. Near criticality, a composite scalar state plays an essential tole in the effective dynamics.

(1) perminem address: Fermilut MS 406, P.O. Bux $5(0)$, Aatavin, IL 60510)

(2) on sabbatical leave: Department of Physics. Purdue University, West Lafayctte, IN 47907 $\therefore$
Quantum electrodynamics has often been used as a convenient laboratory for the study of various properties of quantum field theory. It serves as a model for more complex gauge theories with slowly running coupling constants. Recently there has been considerable interest in the nonperturbative phase structure of gauge theories and the dynamical nature of spontaneous symmetry breaking which may have implications from models of electroweak symmetry breaking to new states of matter in quantum electrodynamics. Anomalous scaling behavior provides a direct indication of the nontrivial nature of the continuum quantum field theory.

In describing the critical behavior associated with a spontaneous symmetry breaking. it is useful and conventionial to introduce various critical exponents ${ }^{(1)}$ which characterize the transition between different phases possessing distinct symmetry properties. One such exponent relates the symmetry breaking order parameter to an externally controlled explicit symmetry breaking variable which can be coupled to it in the underlying theory. For example, in the case of dynamical chiral symmetry breaking, a useful order parameter is the vacuum condensate, $\langle\bar{\psi} \Psi\rangle$. while a soft explicit chiral symmetry breaking is provided by the fermion mass term, $\mu_{0} \bar{\Psi}$. The critical exponent. $\delta_{\mathrm{Cr}}$. is then introduced via the critical scaling law,

$$
\langle\bar{\psi} \Psi\rangle_{\mathrm{criticat}} \approx \mu_{0}\left(1 / \mathrm{C}_{\mathrm{Cr}}\right)
$$

where the $\mu_{0}$ dependence is computed at the critical point.

Since dynamical symmetry breaking is an inherently non-perturbative 
phenomenon, the techniques available for its study are very limited. One such approach which offers a great deal of promise involves lattice computer simulations'2). Although present technology allows for only a limited number of results to be reliably extracted, one such quantity is the critical exponent. $\delta_{c r}$. This follows since $\langle\bar{\psi} \psi\rangle$ can be measured as a function of the gauge coupling constant in both the absence and presence of the explicit symmetry breaking parameter, $\mu_{0}$ When $\mu_{0}$ is set to zero. a nontrivial value for $\langle\bar{\psi} \psi\rangle$ is found for the gauge couplings in a range larger than a critical coupling. At the critical coupling, the order parameter, $\langle\bar{\Psi} \Psi\rangle$, is expected to vanish without symmetry breaking. When $\mu_{0}$ is turned on. $\langle\bar{\psi} \Psi\rangle$ will be nontrivial at the critical coupling and its value there allows for the extraction of the critical exponent. $\delta_{\mathrm{Cr}}$.

This critical exponent can be analytically calculated in various field theoretic models. In the case of QCD, the critical coupling vanishes so that the critical theory is a free field theory for which $\delta_{\mathrm{Cr}}=1$. On the other hand, for the case of mean field theory, the critical exponent is given by $\delta_{\mathrm{Cr}}=3$. Another model which exhibits dynamical chiral symmetry breaking and provides a useful laboratory for the study of certain features of gauge field theory is ladder QED'3,11. We will consider a model which includes chirally invariant four fermion interactions as they can play an important role in the critical behavior's 1

The model is described by the Lagrangian, . .

$$
L=\bar{\Psi}\left(\mid \gamma \cdot D-\mu_{0}\right) \Psi+\left(G_{0} / 2\right) \cdot\left|(\bar{\Psi} \psi)^{2}+\left(\bar{\Psi} \mid \gamma_{5} \psi\right)^{2}\right|
$$

and corresponds to a gauged Nambu-Jona-Lasinio (NJL) model. Treating the gauge interaction in the quenched, planar (ladder) approximation, the model was shown to exhibit a line of critical points separating the chirally broken phase from the symmetric one. The phase diagram in the two coupling constant space is displayed in Figure 1.

In the ladder model, the critical line may be computed ${ }^{(6)}$ and is given b y

$$
G \cdot\left(\alpha / \alpha_{c}\right)=\left(1+\sqrt{1-\alpha / \alpha_{c}}\right)^{2}
$$

where $G=G_{0} \cdot\left(\Lambda^{2} / \pi^{2}\right) \cdot\left(\alpha_{c} / \alpha\right)$ with $\Lambda$ being an ultraviolet momentum cutoff and the gauge coupling constant is in the range, $0<\alpha_{<} \alpha_{c}=\pi / 3$. The point, $\alpha=0$. corresponds to the ordinary NJL model' ${ }^{13}$. In the space above the critical line, the chiral symmetry is dynamically broken. When $\alpha>\alpha_{c}$. the gauge interaction alone is sufficient to trigger the symmetry breaking. while for $0<\alpha<\alpha_{c}$. an additional attractive (G>1) fout fermion interaction is required for the phase transition to occur.

For this range of $\alpha$ values, the fermion condensate takes the form's ,

$$
\begin{aligned}
\langle\bar{\Psi} \Psi\rangle=\left(\tilde{\mathrm{A}} / 4 \pi^{2}\right) \cdot \Lambda \Sigma_{0}^{2} \cdot\left(\alpha_{c} / \alpha\right) \cdot \mid(1-1 / \omega) \cdot\left(\Lambda \mathrm{e}^{\delta / \Sigma_{0}}\right)^{\omega} \\
\left.+(1+1 / \omega) \cdot\left(\Lambda \mathrm{e}^{\delta / \Sigma_{0}}\right)^{-\omega}\right)+\ldots
\end{aligned}
$$


where $\omega=\sqrt{1-\alpha / \alpha_{C}}$ and $\Sigma_{0}=\Sigma(0)$ is the dynamically generated fermion mass scale. $\quad A=\tilde{A}(\alpha)$ and $\delta=\delta(\alpha)$ are parameters of the fermion selfenergy function, $\Sigma(p)$, which is the solution of the Schwinger-Dyson equations of the quenched, planar theory with fixed coupling constant, $\alpha$. $\Sigma(p)$ has the asymptotic expansion $(p \rightarrow \infty)$

$$
\Sigma(p) \rightarrow \tilde{A} \cdot\left(\Sigma_{0}^{2} / p\right) \cdot(1 / 2 \omega) \cdot\left\{\left(p e^{\delta} / \Sigma_{0}\right) \omega-\left(p e^{\delta / \Sigma_{0}}\right)^{-\omega}\right\}+\cdots
$$

In addition, the full fermion bare mass parameter is obtained as

$$
\begin{aligned}
m_{0} & =\mu_{0}-G_{0} \cdot\langle\bar{\Psi} \Psi\rangle \\
& =(\widetilde{A} / 4) \cdot\left(\Sigma_{0}^{2} / \Lambda\right) \cdot\left[(1+1 / \omega) \cdot\left(\Lambda e^{\delta / \Sigma_{0}}\right)^{\omega}+(1-1 / \omega) \cdot\left(\Lambda \mathrm{e}^{\delta / \Sigma_{0}}\right)^{-\omega}\right]
\end{aligned}
$$

The fermion mass scale, $\Sigma_{0}$. is determined from the gap equation

$$
\begin{aligned}
\mu_{0} \cdot \Lambda=(1 / 4) \cdot \tilde{A} \cdot \Sigma_{0}^{2} & +[(1-G) / \omega+(1+G)] \cdot\left(\wedge e^{\left.\delta / \Sigma_{0}\right)^{\omega}}\right. \\
+ & {[-(1-G) / \omega+(1+G)] \cdot\left(\Lambda e^{\left.\left.\delta / \Sigma_{0}\right)^{-\omega}\right)}\right.}
\end{aligned}
$$

which follows from combining Eq.(4) and Eq.(6). Along the critical line defined by Eq.(3), the coefficient of the first tetm on the right hand side precisely vanishes so that the $\mu_{0}$ dependence of $\Sigma_{0}$ is given by $\Sigma_{0} \rightarrow$ $\mu_{0} 1 /(2+\omega)$. Substituting into the expression for the fermion condensate, then gives the critical scaling relation

$$
\langle\bar{\Psi} \Psi\rangle_{\text {critical }} \rightarrow \mu_{0}(2-\omega) /(2+\omega)
$$

from which we extract the critical exponent,

$$
\delta_{c r}=(2+\omega) /(2-\omega)=\left(2+\sqrt{1-\alpha_{\alpha}}\right) /\left(2-\sqrt{1-\alpha_{c} / \alpha_{c}}\right)
$$

For $\alpha=0$. this gives the ordinary NJL model and the mean field value of $\delta_{\mathrm{cr}}=3$, while as $\alpha \rightarrow \alpha_{\mathrm{c}}=\pi / 3$, we find $\delta_{\mathrm{cr}} \rightarrow 1$.

Now consider fixing the four fermion coupling to be some particular value in the range $G>1$ while varying $\alpha$ over the entire range $0<\alpha<\alpha_{C}$. For this value of $G, \alpha$ will approach its critical value, $\alpha^{*}=4 \cdot G /(1+G)^{2}$. where the critical line is encountered. Here the critical exponent will be given by $\delta_{c r}=(3 \cdot G+1) /(G+3)$. On the other hand, away from the critical line, the gap equation is dominated by the first term on the RHS of. Eq.(7). The $\mu_{0}$ dependence of $\langle\vec{\psi} \Psi\rangle$ is obtained by combining Eq.(4) and Eq.(7) yielding $\langle\bar{\psi} \Psi\rangle \approx \mu_{0}$ far from the critical coupling. Thus if the $\mu_{0}$ dependence of $\langle\vec{\Psi} \Psi\rangle$ over the entire range $0<\alpha<\alpha_{C}$ is parameterized as $\langle\bar{\Psi} \psi\rangle \rightarrow\left(A \cdot \mu_{0}(1 / 6)+C\right)$ with $A$ and $C$ being $\mu_{0}$ independent functions of $\alpha$, then the exponent $\delta$ exhibits a sharply peaked behavior attaining its maximum at criticality ${ }^{(8)}$. The form of $1 / 8$ as a function of $\propto$ for fixed $G$ $(=7.873)$ is sketched in Fig.(2). The points represent the calculation in ladder QED using Eq.(4) and Eq.(7). The critical coupling is extracted as, $\alpha^{*} / \alpha_{c}=0.40$, and corresponds to $\delta_{c r} \approx 2.27$. Although this 
parameterization produces the correct behavior both at the critical point and far from criticality, it fails to properly describe the approach to the critical point. Thus, while Fig.(2) can be used to extract the values of $\alpha^{\text {* }}$ and $\delta \mathrm{cr}$. the particular shape of the curve is not an appropriate fit to the near critical behavior. Alternatively, we can fit the $\mu_{0}$ dependence of $\langle\bar{\psi} \Psi\rangle$ as $\langle\bar{\psi} \Psi\rangle \rightarrow A \cdot \mu_{0}^{(1 / \delta)}$ to extract a different value for $\delta$. With this definition, we plot, in Fig.(3), the exponent $1 / 8$ as a function of $\beta=1 / 4 \pi \alpha$ for two distinct values of the explicit bare mass parameter, $\mu_{0}$. The arrow indicates the position of the critical coupling as determined from Fig.(2). Points to the left of the arrow correspond to the chirally broken phase. The vanishing of the exponent $1 / 8$ in this phase reflects the presence of a non-trivial chiral condensate independent of $\mu_{0}$. On the other hand, in the symmetric phase to the right of the artow, a nonvanishing value for $\langle\bar{\psi} \Psi\rangle$ is produced only if $\mu_{0}$ is nonzero. Once again, this fitting fails to accurately model the form of $\langle\bar{\psi} \psi\rangle$ except at criticality and far from the critical point. A more accurate procedure to parameterize the order parameter dependence on $\mu_{0}$ near the critical point follows from the form of the effective potential which we shall obtain subsequently.

In the comparable lattice simulations ${ }^{191}$. One typically starts with pure quenched QED without explicit four-fermion interactions and measures $\langle\bar{\psi} \Psi\rangle$ as a function of the lattice value of $\alpha$. . However, it should be expected that a certain amount of four-fermion interaction will be induced as an artifact of the lattice. The amount of induced coupling depends on the particular lattice regularization employed. Thus the pure lattice QED probes a specific trajectory in the larger space of coupling constants that included the four-fermion interactions. The flow in coupling constant space will not be the simple $G=0$ or $G=$ fixed behavior we have just considered in the ladder QED model. Nonetheless, a particular amount of four-fermion coupling will be induced as a function of $\alpha$ lattice so that a typical flow might have the behavior shown in Fig.(4). Such a flow will take the theory to the critical line for a certain value of $\propto$. The particular value of $\alpha$ depends on the induced four-fermion couplings and therefore the specific lattice regularization. The sharply peaked behavior of the exponent, 8, as a function of $\alpha$ is expected in the quenched, planar QED due to the anomalous dimensions which control the approach to the critical line. This behavior should also be reflected in the lattice simulations if the critical coupling seen there is related to the presence of the critical line in a larger space of couptings than explicitly probed by the simulation. Indeed, the latest simulations of Ref.(9) seem to indicate a value of $\delta_{c r} \approx$ 2.2 which is less than the mean field value, $\delta_{c r}=3$ and would correspond to calculations in the ladder theory with $\alpha \approx \alpha^{*}=0.4 \cdot \alpha_{\mathrm{c}}$

To provide a field theoretic description of the critical behavior, one must include all physically relevant degrees of freedom. In quenched, planar QED. a composite scalar degree of freedom plays a special role in the critical behavior. As the four-fermion coupling gets tuned to near the critical line, the scalar must be considered an independent, propagating degree of freedom with a mass of order the fermion mass scale, $\Sigma_{0}$. The interactions of this scalar degree of freedom become relevant near 


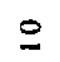

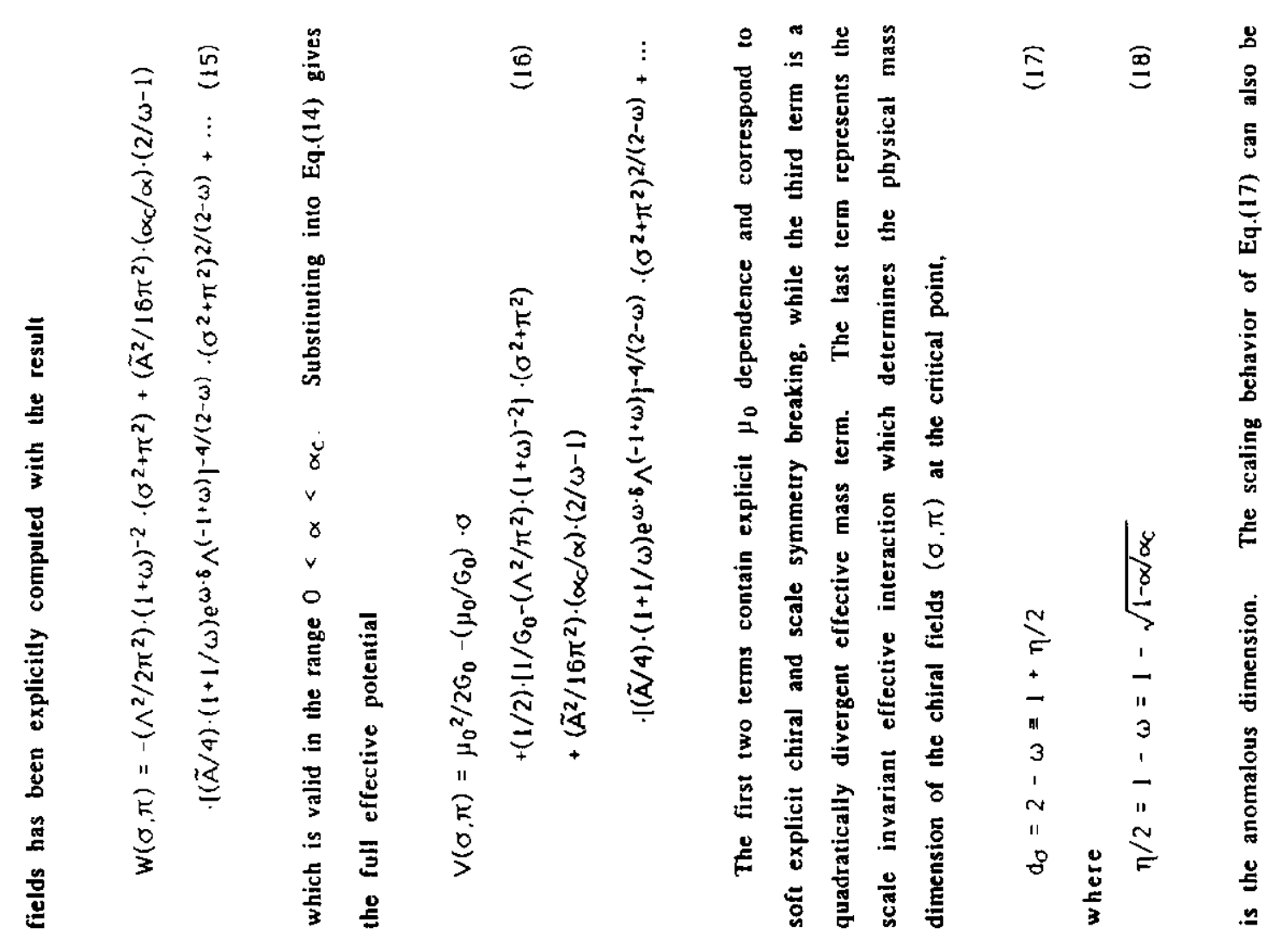

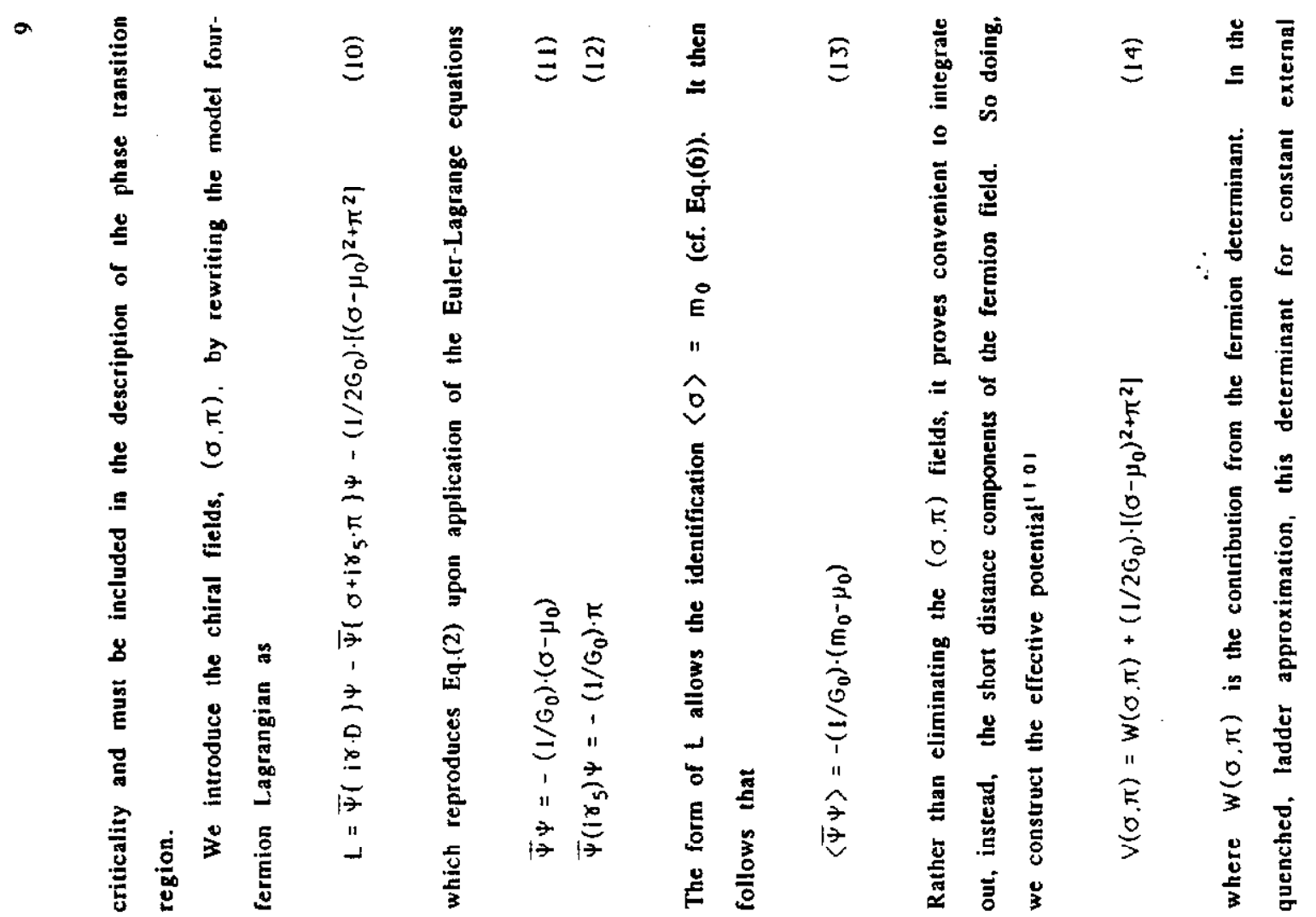


obtained directly"ll using the methods of Ref.(12) where the renormalization constant of the bare mass, $\mu_{0}$, was calculated along the critical line.

Using the effective potential, the origin of the fine tuning of the fourfermion coupling becomes apparent. Unless $G$, is tuned to be near the critical line, the quadratically divergent mass term will dominate the potential and the chiral fields will not propagate. When tuned to the critical line, this mass term precisely vanishes and the minimum of the potential gives

$$
\Sigma_{0} \mid \operatorname{crit|cal}=\left((1-\omega) e^{\omega \cdot 6} \Lambda^{(1+\omega)} / \tilde{A}\right\}^{1 /(2+\omega)} \cdot \mu_{0}^{1 /(2+\omega)}
$$

To secure the critical exponent, $f_{c r}$. we use Eq.(6) and Eq.(19) with Eq.(13) to give the critical scaling law

$$
\begin{aligned}
& \left.\langle\bar{\Psi} \Psi\rangle\right|_{\text {critical }}=\left(\wedge^{2} / 4 \pi^{2}\right) \cdot(1 / \omega) \cdot \mu_{0} \\
& -\left(\tilde{A} / 4 \pi^{2}\right) \cdot\left(\alpha_{c} / \alpha\right) \cdot(1 / \omega) \cdot\left[(1-\omega) e^{\omega \cdot \delta} \Lambda^{(1+\omega) / \tilde{A})^{4 /(2+\omega)} \cdot \mu_{0}(2-\omega) /(2+\omega)}\right.
\end{aligned}
$$

which implies the form for $\delta_{c r}$ given in Eq. $(9), \delta_{c r}==(2+\omega) /(2-\omega)=$ $\left(2+\sqrt{1-\alpha / \alpha_{c}}\right) /\left(2-\sqrt{1-\alpha / \alpha_{c}}\right)$.

In addition to the critical exponent. $\delta_{\mathrm{cr}}$. the effective potential can also be used to extract other critical exponents for the ladder theory. These exponents are defined to quantify the approach to criticality of the order parameter, the vacuum potential, and the scalar mass. That is, they are designed to measure the dependence of these quantities on $\Delta G=$ $G \cdot\left(\alpha / \alpha_{C}\right)-(1+\omega)^{2}$ which corresponds to the deviation from criticality. In particular, the critical exponents $\tilde{\alpha}, \mathfrak{b}, \gamma, v$ are defined as

$$
\begin{aligned}
& V(\langle\sigma\rangle, 0) \rightarrow(\Delta G)^{2-\tilde{\alpha}} . \quad \mu_{0}=0 \\
& \langle\bar{\Psi} \psi\rangle \rightarrow(\Delta G)^{\beta}, \quad \mu_{0}=0 \\
& \partial_{\mu_{0}}\langle\bar{\Psi} \Psi\rangle \rightarrow(\Delta G)^{-\gamma}, \quad \mu_{0} \rightarrow 0 \\
& m_{\sigma} \rightarrow(\Delta G)^{v}, \quad \mu_{0} \rightarrow 0
\end{aligned}
$$

where we have used $\tilde{\alpha}$ instead of $\propto$ to avoid confusion with the gauge coupling constant.

From an examination of the gap equation, or equivalently by minimizing the effective potential with respect to $m_{0}$. it follows that near the critical line the dynamically generated fermion mass scale has the dependence

$$
\begin{aligned}
& \Sigma_{0} \rightarrow(\Delta G)^{1 / 2 \omega}, \quad \mu_{0}=0 \\
& \partial_{\mu_{0}} \Sigma_{0}(2-\omega) \rightarrow(\Delta G)^{-1}, \quad \mu_{0} \rightarrow 0
\end{aligned}
$$

Combining this behavior with the known $\Sigma_{0}$ dependence of $m_{0}$ as given by Eq.(6) yields 


$$
\begin{aligned}
& m_{0} \rightarrow(\Delta G)^{(2-\omega) / 2 \omega}, \quad \mu_{0}=0 \\
& \partial_{\mu_{0}} m_{0} \rightarrow(\Delta G)^{-1}, \quad \mu_{0} \rightarrow 0
\end{aligned}
$$

The exponents $\beta$ and $\gamma$ can be obtained directly using Eq.(13),

$$
\begin{aligned}
& \beta=(2-\omega) / 2 \omega=\left(2-\sqrt{1-\alpha / \alpha_{c}}\right) / 2 \sqrt{1-\alpha / \alpha_{c}} \\
& \gamma=1
\end{aligned}
$$

Next we use Eq.(17) in the chiral limit $\left(\mu_{0}=0\right)$ to find the scaling behavior of the full effective potential, $V\left(m_{0}, 0\right) \rightarrow\left(m_{0}\right)^{4 /(2-\omega)}$ which in turn gives the exponent $\tilde{\alpha}$.

$$
\tilde{\alpha}=2 \cdot(\omega-1) / \omega=2 \cdot\left(\sqrt{1-\alpha / \alpha_{C}}-1\right) / \sqrt{1-\alpha / \alpha_{C}}
$$

Finally the exponent $v$ is secured by noting that in the scaling region

$$
m_{\sigma} \approx \Sigma_{0} \rightarrow(\Delta G)^{(1 / 2 \omega)}
$$

so that

$$
\nu=1 / 2 \omega=1 / 2 \sqrt{1-\alpha / \alpha_{c}}
$$

Combining the expressions for the various critical..exponents, it follows that usual scating relations,

$$
\begin{aligned}
& 2 \cdot \beta+\gamma=2-\tilde{\alpha} \\
& 2 \cdot \beta \cdot \delta-\gamma=2-\tilde{\alpha} \\
& \gamma=\nu \cdot(2-\eta) \\
& 4 \cdot \nu=2-\tilde{\alpha}
\end{aligned}
$$

are indeed satisfied.

The effective potential computed in Eq.(16) can provide a way to properly parameterize the approach to the critical point. Vacuum properties are obtained by minimizing this potential which yields,

$$
\begin{aligned}
& \mu_{0} / G_{0}=\left[1 / G_{0}-\left(\Lambda^{2} / \pi^{2}\right) \cdot(1+\omega)^{-2}\right] \cdot m_{0}+\left(1 / 4 \pi^{2}\right) \cdot \tilde{A}^{2} \cdot\left(\alpha_{c} / \alpha\right) \\
& \cdot(1 / \omega) \cdot\left[(\tilde{A} / 4) \cdot(1+1 / \omega) e^{\omega \cdot \delta} \Lambda^{(-1+\omega)}\right\}^{=4 /(2-\omega)} \cdot\left(m_{0}\right)(2+\omega) /(2-\omega)
\end{aligned}
$$

with $m_{0}$ related to $\langle\bar{\Psi} \psi\rangle$ via Eq.(13). Guided by this form obtained in the explicit ladder calculation, it follows that an accurate parameterization of the data for quenched QED should take the form,

$$
\mu_{0}=A \cdot\langle\bar{\Psi} \Psi\rangle+B \cdot\langle\bar{\Psi} \Psi\rangle^{\delta}
$$

where $A, B$ and $\delta$ are nonuniversal functions of the coupling constants to be fitted. The normal fitting procedure would require that Eq.(36) be numerically inverted to express $\langle\bar{\psi} \psi\rangle$ as a function of the parameters $\mathrm{A}$. B. $\delta$ and $\mu_{0}$. We note that larger values of $\mu_{0}$, and/or larger values of the condensate, may require higher order terms of the effective potential' 10 , 
to get an accurate description of the dependence of $\langle\bar{\psi} \psi\rangle$ on $\mu_{0}$ and $\alpha$

We have seen that the four-fermion interactions play an important role in determining the scaling behavior of quenched, planar QED. This is particularly true at weak gauge coupling where the amount of fourfermion interaction affects the critical value of $\propto$ at the phase transition, the critical line, and therefore the value of the critical indices. However, this sensitivity seems surprising as the four-fermion operators would normally be considered as irrelevant interactions since their effective physical dimension is greater than four. Normally, it should be possible to absorb the effects of the all irrelevant interactions by suitable modifications of the coupling strengths of the relevant interactions 13 , This "theorem" would seem to be violated by the observed critical scaling behavior.

A related aspect of the scaling behavior is the fine tuning which is neccessary to approach the critical limit. Away from the critical line, the four-fermi interactions are, indeed, irrelevant as their effects are suppressed by powers of the cutoff. It is only when the couplings are tuned very close to the critical line that the continuum scaling behavior is seen. The physical picture in the critical region requires that new composite degrees of freedom be introduced and it is the relevant interactions of these composite degrees of freedom which determine the critical behavior. In the case of quenched QED, the fine tuning is expected to produce scalar and pseudoscalar degrees of freedom with chiral invariant interactions as seen by the effective potential of Eq.(16). We now see that the "theorem" on relevant interactions is not violated but we must include the possibility of generating composite degrees of freedom.
( $\sigma, \pi, e t c)$, and their relevant interactions in addition to the dynamics associated with the fermions and gauge interactions.

The above physical picture is needed to understand the structure of the quenched theory where the critical line is presumed to exist with induced four-fermion interactions being present even in the pure gauge theory. In the full unquenched QED, the running of the gauge coupling constant will modify the scaling structure and it is likely that the critical line will disappear being replaced by either a true ultraviolet fixed point or triviality. Even in this case, it may be necessary to introduce the composite degrees of freedom and their effective interactions to properly understand the physics near the continuum limit 14,15 ,

We have shown that irrelevant interactions may play a crucial role in understanding the dynamical structure of gauge theories. These results may have important implications for the proper interpretation of lattice field theory simulations where irrelevant interactions are expected to be generated by the regularization procedure. In the quenched version of lattice QED, only the gauge coupling appears as an explicit parameter. However, one should vicw the simulations as corresponding to theories defined in a large space of possible induced interactions and their couplings. By varying the explicit gauge coupling, the simulation follows a specific trajectory in the space of induced couplings. In most cases, these induced couplings have little effect on the infrared dynamics of the theory. However. in the quenched theory. we have seen that these small induced couplings. $\left(G_{0} \rightarrow O\left(1 / \wedge^{2}\right)\right)$, can play a crucial role in determining the critical couplings and the scaling behavior at the critical point. In interpreting lattice field theory simulations, one must try to determine 
whether the induced interactions can play a significant role in the critical, or near critical behavior seen in the simulations.

We have made an explicit study of the scaling behavior of quenched, planar (ladder) QED. We have shown that small four-fermion interactions can strongly affect the critical behavior of the theory. We have made an explicit calculation of the critical indices along the critical line for $0<\alpha<$ $\alpha_{c}$. To compare with specific lattice simulations, one must be sure to identify the theories at the appropriale value of the gauge coupling constant defined in each theory. The critical indices reflect the anomalous dimensions of the theory and the full set of critical indices should agree if a proper identification is made for the gauge coupling constant. To aid in this comparison, we plot in Fig.(5) the critical exponent, $\delta_{\mathrm{cr}}$, as given in Eq.(9) as a function of the gauge coupling constant, $\alpha / \alpha_{c}$. defined in quenched, planar QED.

The physics near the critical line should be understood in terms of the dynamics of composite degrees of freedom, $(\sigma, \pi)$, which become active through the fine-tuning required to approach the critical line. The formally irrelevant four-fermion interactions are replaced by relevant interactions of the composite degrees of freedom. These results have been obtained for ladder QED and are consistent with the lattice simulations for quenched QED. The nature of the critical line could change in the unquenched version of the theory due to the additional running of the gauge coupling constant. Nevertheless, the rote of the dynamics of the composite states and formally irrelevant interactions near the critical point may be a more general feature of quantum field theory and important in the interpretation of lattice field theory simulations.

\section{Acknowledgements}

We thank Elbio Dagotto for many useful discussions on lattice simulations. V.A.M. thanks John Kogut for enjoyable discussions. We thank the members of the Institute for Theoretical Physics for their hospitality and support.

This research was supported in part by the National Science Foundation under Grant No. PHY89-04035, supplemented by funds from the National Aeronautics and Space Administration, at the University of California at Santa Barbara. This work was also supported in part by the U.S. Department of Energy. S.L. was provided support at Purdue under Grant DE-ACOL-76ER01428 (Task B). 
References

[1] See, for example, D. Amit, "Field Theory, the Renormalization Group and Critical Phenomena", McGraw.Hill (1978).

[2] J.B. Kogut, E. Dagotto and A. Kocic, Phys. Rev. Lett. 60(1988)772; Nuct. Phys. B317(1989)253; Nucl. Phys. B317(1989)271; S. Hands, J.B. Kogut and E. Dagotto, Nucl. Phys. B333(1990)551; S.P. Booth, P.D. Kenway and B.J. Pendleton, Phys. Lett. 228B(1989)175; M. Gockeler, R. Horsley, E. Laermann, P. Rakow, G. Schierholz, R. Sommer and U.J. Wiese, Nucl. Phys. B334(1990)527.

[3] K. Johnson, M. Baker and R. Willey, Phys. Rev. 136(1964)B 1111; Phys. Rev. 163(1967)1699; T. Maskawa and H. Nakajima, Prog. Theor. Phys. 52(1974)1326; Prog. Theor. Phys. 54(1975)860; R. Fukuda and T. Kugo, Nucl. Phys. B 117(1976)250; S.L. Adler and W.A. Bardeen. Phys. Rev. D4(1971)3045.

[4] V.A. Miransky, Nuovo Cim. 20A(1985)149; Sov, Phys. JETP 61(1985)905; P.I. Fomin, V.P. Gusynin, V.A. Miransky and Yu.A. Sitenko, Riv. Nuovo Cim, 6(1983)1.

[5] C.N. Leung, S.T. Love and W.A. Bardeen, Nucl. Phys. B273(1986)649; W.A. Bardeen, C.N. Leung and S.T. Love, Nucl. Phys. B323(1989)493.

[6] K.-I. Aoki, Kyoto Univ. Preprint RIFP 758 (1988); K.-I. Kondo, H. Mino, and K. Yamawaki, Phys. Rev. D39(1989)2430; T. Appelquist, M. Soldate. T. Takeuchi and L.C.R. Wijewardhana, in "Proceedings of the $12 \mathrm{th}$ Johns Hopkins Workshop on Current Problems in Particle Theory", ed. by G. Domokos and S. Kovesi-Domokos, World Scientific (1988); M. Inoue, T Muta and T. Ochiumi. Mod. Phys. Lett. A4(1989)605.

[7] Y. Nambu and G. Jona-Lasinio, Phys. Rev. 122(1961)345

[81 That the scaling fits to the condensate, as shown in Fig.(2), can be used to determine the critical coupling was emphasized to us by E. Dagotto, private communication.

191 A. Kocic, S. Hands, J. Kogut, and E. Dagotto, SBITP Preprint NSF-ITP-90 $62(1990)$.
[10] W.A. Bardeen and S.T. Love, in preparation

[11] V.A. Miransky, in preparation.

[12] V.A. Miransky and K. Yamawaki, Mod. Phys. Lett. A4(1989)129.

[13] K.G. Wilson and J.B. Kogut, Phys. Rept. 12C(1974)77.; T. Appelquist and J. Carrazone. Phys. Rev. DU1(1975)2856.

[14] for explicit applications to top condensate models, see: V.A. Miransky, M. Tanabashi and K. Yamawaki, Phys. Lett. B221(1989)177, W.A. Bardeen, C.T. Hill and M. Lindner, Phys. Rev. D41(1990)1647.

[15] for applications to technicolor models, see: T. Appelquist, T Takeuchi, M. Einhosn and L.C.R. Wijewardhana, Phys. Lelt. 220B(1989)223; T. Takeuchi, Phys. Rev. D40(1989)2697: V.A. Miransky, M. Tanabashi and K. Yamawaki, Phys. Lett. 221(1989)177; Mod. Phys. Lett. A4(1989)1043; R.S. Chivukula, A.G. Cohen and K. Lane, SBITP Preprint, NSF-ITP-90-52. 


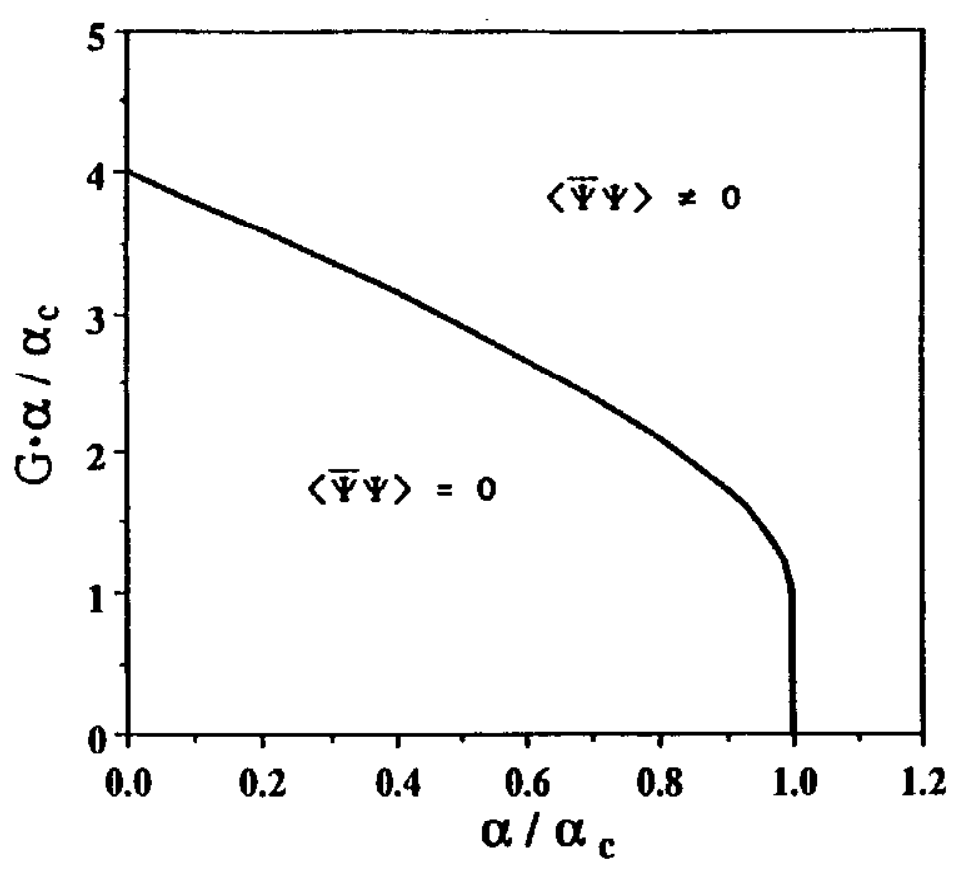

Fig. 1. Chiral symmetry phase diagram in coupling constant space for ladder QED. The critical curve is given by $G \cdot\left(\alpha / \alpha_{c}\right)=\left(1+\sqrt{1-\alpha / \alpha_{c}}\right)^{2}$.

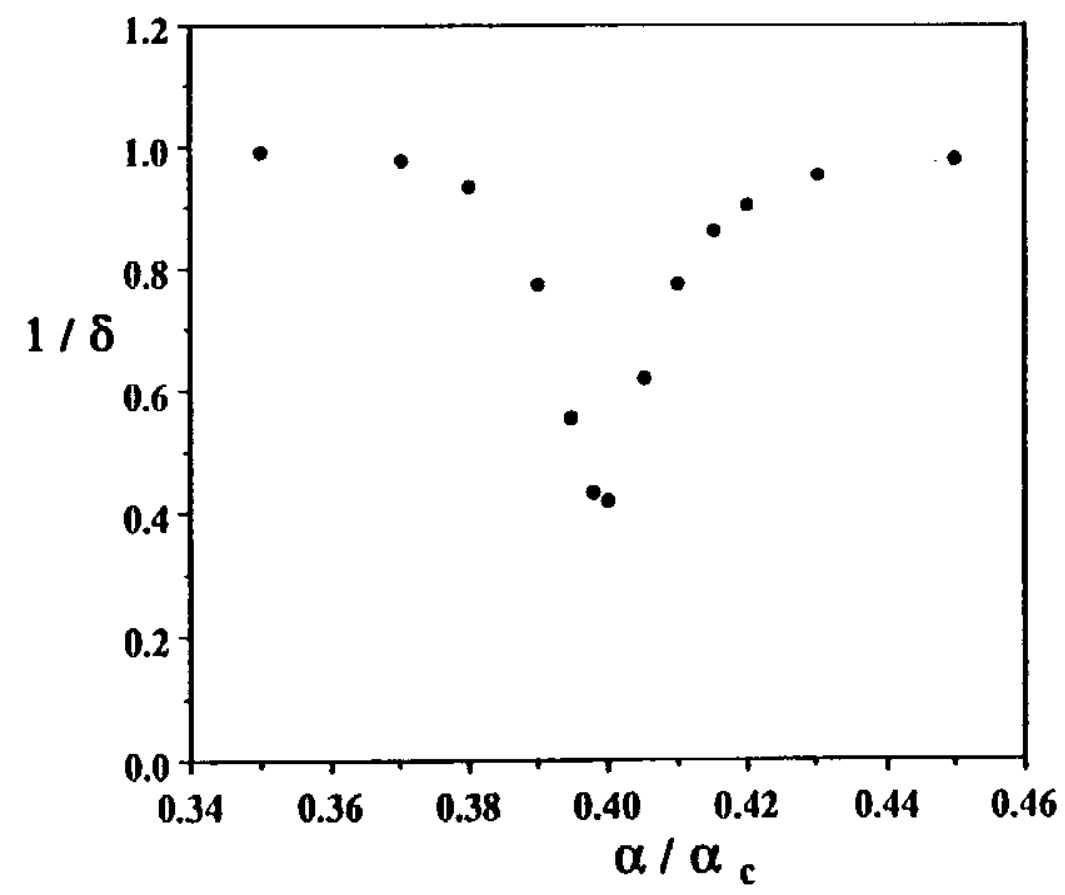

Fig. 2. The critical exponent $\delta$ as a function of $\alpha / \alpha_{c}$ defined using the functional form, $\langle\vec{\psi} \psi\rangle=A \cdot \mu_{0}(1 / \delta)+C$. 

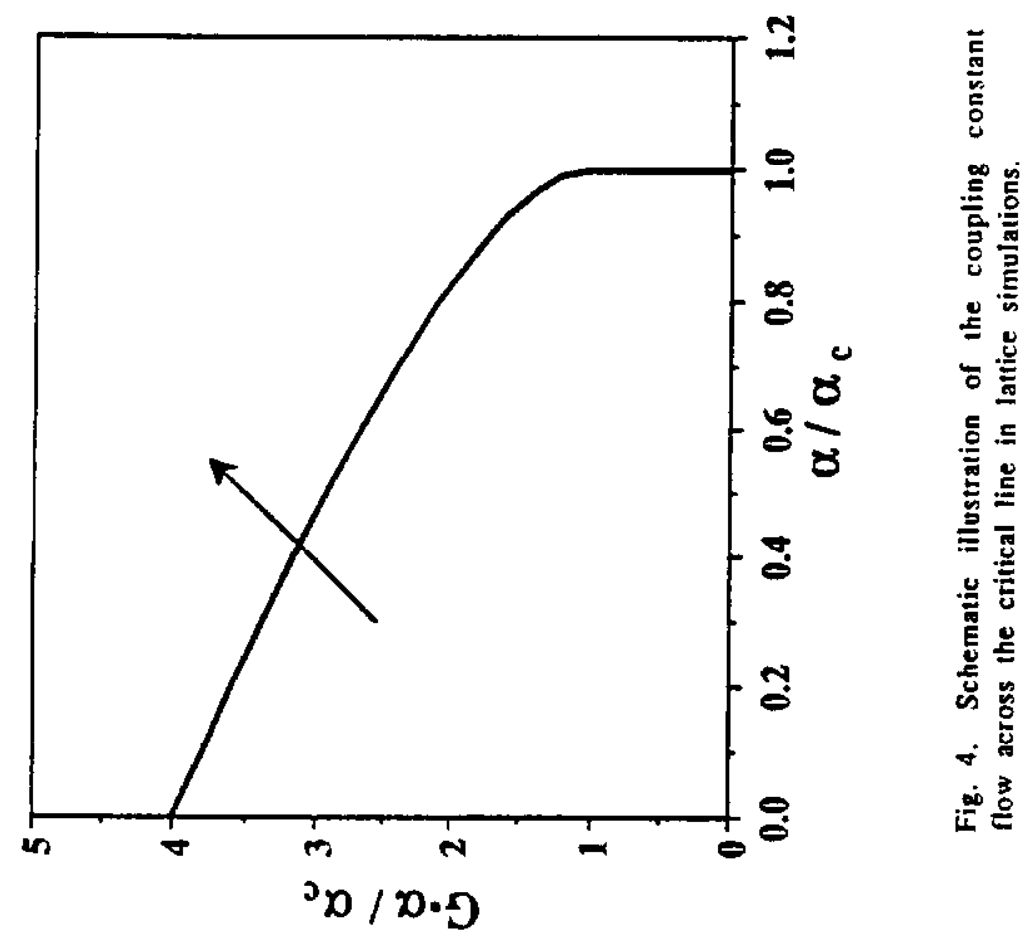

2
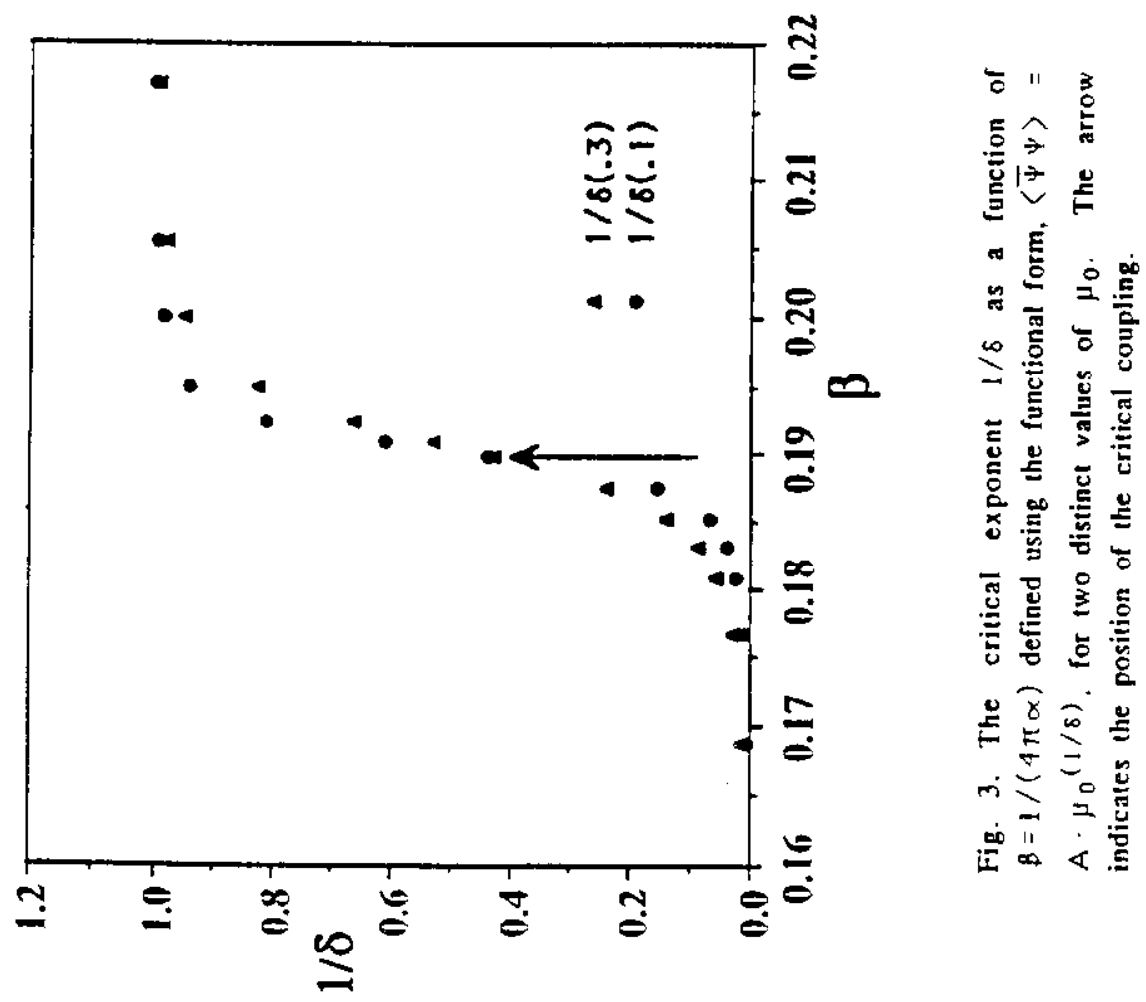
N

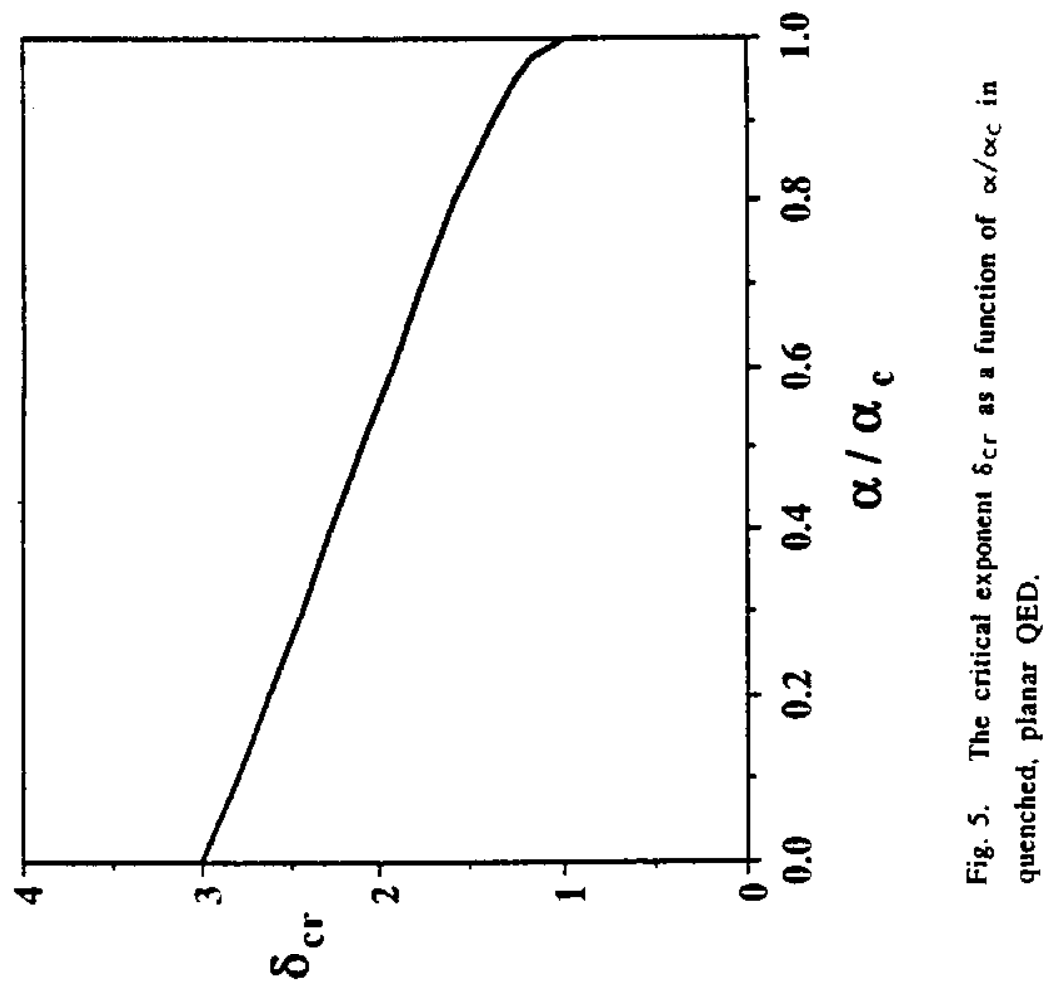

